\title{
Remoción de formas parasitarias intestinales en una laguna facultativa de estabilización en Lima, Perú
}

\author{
José O. lannacone ${ }^{1}$
}

\begin{abstract}
Removal of intestinal parasitic forms in a facultative stabilization pond in Lima, Peru. The effectiveness of a waste stabilization pond, to remove intestinal helminth eggs and protozoan cysts was studied in San Martin de Porres, Lima, Peru. From September to October 2000 four collections of samples were performed from raw domestic wastewater and from contends of primary, secondary and tertiary ponds. A comparative study of performance of the concentrations methods of Ritchie and Faust was also done. The overall average removal of parasitic forms was as follow: $69.37 \%$ from raw water and primary pond; $48.34 \%$ from primary to secondary pond; $85.45 \%$ from secondary to tertiary pond and $97.69 \%$ from raw water to tertiary pond. There was no significant difference in the quantitative performance of Ritchie and Faust methods. Giardia duodenalis (Lambl, 1859) Alexeieff, 1914, Entamoeba coli (Gras, 1879) Casagrandi \& Barbagallo, 1895 and Ascaris lumbricoides (Linnaeus, 1758 ) were detected with both methods. Three other parasites were detected only by Ritchie method: Taenia sp., Rodentolepis nana (von Siebold, 1852) Spasskii, 1954 and Trichuris trichiura (Linnaeus, 1771) Stiles, 1901 and only Faust detected Isospora belli Wenyon, 1923. Overall number of protozoa cysts was higher than the number of helminth eggs. At the end, wastewater had a concentration of 1.5 parasitic forms $\mathrm{L}^{-1}$ what is considered inappropriate for ultimate use in agriculture by current standards. KEY WORDS. Agricultural land, Ascaris lumbricoides, domestic sewage, Entamoeba coli, Giardia duodenalis, Peru
\end{abstract}

Las aguas residuales domésticas son portadoras de una gran diversidad de flora bacteriana de origen fecal (TORANZOS 1991). Además son receptoras de formas parasitarias de protozooos y de helmintos. Muchos de estos microorganismos son agentes etiológicos de enfermedades de importancia en Salud Pública (CARRINGTON et al. 1991; ZAPATA et al. 1991).

Entre los protozoos encontrados en las aguas residuales tenemos al flagelado Giardia duodenalis (Lambl, 1859) Alexeieff, 1914 causante de diarreas en niños y adultos a nivel mundial y a la Entamoeba histolytica Schaudinn, 1903 causante de la disentería amebiana (ROSE et al. 1988; JAKUBOWSKI et al. 1991; ROSE \& GERBA 1991; CHAvEZ et al. 1994). Además en las aguas residuales, se pueden encontrar quistes del protozoo no patogénico Entamoeba coli (Gras, 1879) Casagrandi \& Barbagallo, 1895 y es común encontrar huevos de diversos helmintos parásitos como Taenia saginata (Goeze, 1782) Weinland, 1858, Taenia solium Linnaeus, 1758, Necator americanus (Stiles, 1902) Stiles, 1906, Ascaris lumbricoides (Linnaeus, 1758), Trichuris trichiura (Linnaeus, 1771) Stiles, 1901 y Rodentolepis nana (von Siebold, 1852) Spasskii, 1954 (SHUVAL et al. 1985; SÁNCHEZ 1991; HOWARD et al. 2001).

1) Laboratorio de Ecofisiología, Facultad de Ciencias Naturales y Matemáticas, Universidad Nacional Federico Villarreal. Calle San Marcos 383, Pueblo Libre, Lima 21, Perú.

E-mail: joselorena@terra.com

Revta bras. Zool. 19 (4): 1033 - 1041, 2002 
Una de las formas de tratar las aguas residuales de origen doméstico con el fin de disminuir su carga parasitológica de origen fecal para su posterior uso en agricultura, para uso municipal, en el ámbito forestal, en parques recreacionales, en acuicultura y para el uso industrial, es el empleo de las denominadas lagunas de estabilización u oxidación (WATSON et al. 1983; MARA \& SILVA 1986; DE LOS RIOS 1998; SMITH 1999). En estos sistemas de tratamientos de aguas residuales, los análisis biológicos y fisicoquímicos son una parte importante para el diseño, operación y mantenimiento de estas plantas de tratamiento, por lo que es aconsejable realizar muestreos continuos con el fin de estimar la eficiencia de la remoción de la parasitofauna, para así determinar su posterior adecuado reuso (ESPINOZA 1998; HABBARI et al. 2000).

BOUHOUM \& SCHWARTZBROD (1989) han señalado que al examinar diferentes tipos de aguas residuales, existen diferencias en el número de especies concentradas e identificadas dependiendo de la técnica de concentración de parásitos empleada.

De esta forma el objetivo del presente estudio fue evaluar la eficiencia de la remoción de huevos de helmintos y quistes de protozoos intestinales en una Laguna facultativa de estabilización de aguas residuales domésticas en el distrito de San Martín de Porres, Lima, Perú, empleando comparativamente dos técnicas diferentes de concentración de parásitos.

\section{MATERIAL Y MÉTODOS}

\section{Área de estudio}

Las lagunas facultativas de oxidación de la Universidad Nacional de Ingeniería (UNI) se encuentran ubicadas en el km 11,5 de la Av. Túpac Amaru, distrito de San Martín de Porres, Lima, Perú. El caudal de ingreso (Q) promedio de las aguas residuales crudas es de $10 \mathrm{~L} \mathrm{~s}^{-1}$ proveniente del distrito de Independencia. La Laguna primaria tiene un área aproximada de $100 \mathrm{~m} \mathrm{x} 50 \mathrm{~m}$ y las lagunas secundarias y terciarias aproximadamente de $50 \mathrm{~m} \times 50 \mathrm{~m}$. La profundidad para todos los casos varió entre 1,5 m a $2 \mathrm{~m}$ (SENCICO 1996). Con relación a la DBO soluble 5,20 promedio durante el estudio se observaron los siguientes valores en cada una de las fases del tratamiento: Crudo: $67 \mathrm{mg} \mathrm{L}^{-1}$; Primaria: $15 \mathrm{mg} \mathrm{L}^{-1}$; Secundaria: $19 \mathrm{mg}$ $\mathrm{L}^{-1}$ y Terciaria $15 \mathrm{mg} \mathrm{L}^{-1}$. En el caso de los sólidos suspendidos totales se tuvieron los siguientes valore promedios: Crudo: $270 \mathrm{mg} \mathrm{L}^{-1}$, Primaria: $96 \mathrm{mg} \mathrm{L}^{-1}$; Secundaria: $111 \mathrm{mg} \mathrm{L}^{-1}$ y Terciaria: $103 \mathrm{mg} \mathrm{L}^{-1}$.

\section{Muestreos}

Durante septiembre a octubre del 2000 , se realizaron cuatro evaluaciones quincenales de las aguas residuales domésticas en cuatro puntos de muestreo: crudo, laguna primaria, secundaria y terciaria en busca de formas parasitarias intestinales. Para la colecta de las muestras parasitológicas a partir de estas aguas residuales domésticas, se emplearon frascos de polietileno con capacidad de $4 \mathrm{~L}$. Para el agua residual cruda se tomó $1 \mathrm{~L}$, para la laguna primaria $2 \mathrm{~L}$ y para la laguna secundaria y terciaria $4 \mathrm{~L}$, respectivamente. Las muestras se almacenaron en refrigeración a 
una temperatura de $4^{\circ} \mathrm{C}$ por no más de 48 h después de su colección. En todos los puntos, se tomaron las muestras por duplicado para ser empleadas en cada una de las dos técnicas de concentración parasitológicas utilizadas. La toma de las muestras se realizó en todos los casos de 10:00 am a 11:00 am.

\section{Técnicas parasitológicas}

Consistieron en tomar las muestras de aguas residuales colectadas de cada uno de los cuatro puntos de muestreo en su respectiva evaluación y su posterior centrifugación en tubos de centrífuga de $250 \mathrm{ml}$ a $2300 \mathrm{rpm}$ durante dos minutos, decantando el sobrenadante. Este proceso de centrifugación fue realizado por lo menos tres veces, hasta la obtención de un sobrenadante casi libre de materia en suspensión (AYRES \& MARA 1997). Los sedimentos obtenidos por este procedimiento se utilizaron en conjunto para la técnica de concentración de flotación de Faust y de sedimentación de Ritchie (OMS 1992; APHA 1995).

Flotación de Faust. Los sedimentos obtenidos fueron colocados en tubos graduados de centrífuga de $50 \mathrm{ml}$. Posteriormente, se le adicionó una solución de sulfato de $\mathrm{Zinc}\left(\mathrm{ZnSO}_{4} .7 \mathrm{H}_{2} \mathrm{O}\right.$ al 33,1\%, p/v), con una gravedad específica de 1,20 y se le realizó una centrifugación a 2300 rpm durante dos minutos. Luego, se colocó una laminilla cubreobjeto sobre la abertura del tubo de centrífuga y finalmente se le trasladó a una cámara de Neubauer con una gota de solución yodada de D'Antoni, para su observación y conteo al microscopio a un aumento de 400x.

Sedimentación de Ritchie. A los sedimentos obtenidos, se les adicionó 10 $\mathrm{ml}$ de formalina al $10 \%$ y $5 \mathrm{ml}$ de éter etílico de grado analítico en tubos graduados de centrífuga de $50 \mathrm{ml}$ y se taparon los tubos y se procedió a agitar vigorosamente por 20 s. Luego, se centrifugó a 2000 rpm durante uno minuto. Se eliminó el tapón superior de detritus que se formó en la superficie del tubo de centrífuga con la ayuda de un aplicador de vidrio. Luego se decantó el sobrenadante, a excepción del sedimento. Finalmente, todo el sedimento se mezcló y se transfirió con una pipeta Pasteur a una cámara de conteo de Neubauer con 1 gota de la solución yodada de D'Antoni, cubriéndolo con laminillas cubreobjetos y se observó y se contó en el microscopio a un aumento de 400x (VARGAS 1988).

\section{Análisis de datos}

Se emplearon los siguientes métodos estadísticos: la prueba de t de Student para datos pareados para determinar si existieron diferencias entre el número total de formas parasitarias encontradas por ambas técnicas parasitológicas de concentración e inclusive entre cada una de las etapas de muestreo independientemente; la prueba de ANDEVA para determinar si existían diferencias en el número total de formas parasitarias en las cuatro etapas del tratamiento y entre los cuatro muestreos temporales. Además, esta misma prueba se utilizó para observar si existían diferencias en el número de cada una de las formas parasitarias entre sí y entre cada una de las cuatro etapas del tratamiento (ZAR 1996). El nivel de significancia fue evaluado a alfa $=0,05$ (DANIEL 1993). La terminología ecológica siguió los criterios de BUSH et al. (1997). Se empleó el paquete estadístico SPSS versión 7,5 para Windows ${ }^{\circledR} 95$ para el cálculo de las pruebas estadísticas descriptivas e inferenciales. 


\section{RESULTADOS}

Mediante ambas técnicas de concentración parasitológica se registraron siete especies parasitarias: tres protozoos en fase quística, dos platelmintos y dos nemátodos, estos dos últimos en la fase de huevo (Tabs I y II).

La tabla I, nos muestra el número promedio de formas parasitarias encontradas por la técnica de concentración de flotación de Faust en cada una de las cuatro fases del tratamiento de las aguas residuales domésticas. Se registraron por la técnica de Faust cuatro especies: G. duodenalis, E. coli, Isospora belli Wenyon, 1923 y A. lumbricoides. Los protozoos $G$. duodenalis y $E$. coli se encontraron generalmente en mayor cantidad que los huevos del nemátodo A. lumbricoides en cada una de los cuatro puntos de muestreos en la laguna de estabilización. El orden del porcentaje de remoción en orden descendente de cada una de las formas parasitarias del crudo a la laguna terciaria fue: A. lumbricoides $>I$. belli $>$ G. duodenalis $>E$. coli. No se observó ninguna relación lineal estadísticamente significativa entre el tamaño promedio de la forma parasitaria intestinal en $\mu$ y el porcentaje de remoción total ( $\mathrm{r}$ $=0,77, \mathrm{p}=0,22, \mathrm{n}=4)$, ni tampoco relación logarítmica $(\mathrm{r}=0,77, \mathrm{p}=0,23, \mathrm{n}=4)$.

Tabla I. Promedio de formas parasitarias $\mathrm{L}^{-1}$ encontradas por la técnica de concentración de Flotación de Faust en cada una de las cuatro fases del tratamiento de aguas residuales domésticas en una Laguna de estabilización en Lima, Perú.

\begin{tabular}{lcccccc}
\hline & \multicolumn{5}{c}{ Formas parasitarias } \\
\cline { 2 - 7 } & Tamaño promedio $(\mu)$ & Cruda & Primaria & Secundaria & Terciaria & $\%$ Remoción total \\
\hline Giardia duodenalis & 10 & $19,8 \mathrm{a}$ & $7,70 \mathrm{a}$ & $3,50 \mathrm{ab}$ & $0,57 \mathrm{a}$ & 97,12 \\
Entamoeba coli $^{*}$ & 25 & $21,6 \mathrm{a}$ & $7,30 \mathrm{ab}$ & $4,12 \mathrm{a}$ & $0,63 \mathrm{a}$ & 97,08 \\
Isospora belli $_{\text {Ascaris lumbricoides }}$ & 30 & $10,3 \mathrm{~b}$ & $3,00 \mathrm{c}$ & $1,15 \mathrm{bc}$ & $0,18 \mathrm{~b}$ & 98,25 \\
Prueba de Fisher & 60 & $10,8 \mathrm{~b}$ & $3,62 \mathrm{bc}$ & $1,00 \mathrm{c}$ & $0,18 \mathrm{~b}$ & 98,33 \\
Probabilidad & - & 47,28 & 7,58 & 7,64 & 8,14 & - \\
\hline
\end{tabular}

*) Especie no patogénica. Letras minúsculas iguales en sentido vertical indican que los promedios de formas parasitarias fueron estadisticamente iguales. Prueba de Tukey (SPSS versión 7,5).

La tabla II, nos indica el número promedio de formas parasitarias intestinales registradas por la técnica de sedimentación de Ritchie en los diferentes puntos de muestreo en la laguna de oxidación de San Martín de Porres, Lima, Perú. Las seis especies registradas fueron: $G$. duodenalis, E. coli, Taenia sp., $R$. nana, A. lumbricoides y $T$. trichiura. Nuevamente los protozoos fueron más abundantes que los helmintos. El orden de remoción del crudo a la laguna terciaria fue en el siguiente orden descendente: $R$. nana $=$ Taenia sp. $>$ T. trichiura $>$ A. lumbricoides $>E$. coli $>G$. duodenalis. No se observó ninguna relación lineal estadísticamente significativa entre el tamaño promedio de la forma parasitaria intestinal en $\mu$ y el porcentaje de remoción total $(r=0,75, p=0,08, n=6)$. Aunque numéricamente los helmintos (generalmente de mayor tamaño) registraron mejores remociones que los protozoos (Tab. II). Además, si se encontró una relación logarítmica estadísticamente significativa entre el tamaño de la forma parasitaria y el porcentaje de remoción $(r=0,84$, $\mathrm{p}=0,03, \mathrm{n}=6$ ). 
Tabla II. Promedio de formas parasitarias $\mathrm{L}^{-1}$ encontradas por la técnica de concentración de Sedimentación de Ritchie en cada una de las cuatro fases del tratamiento de aguas residuales domésticas en una Laguna de estabilización en Lima, Perú.

\begin{tabular}{|c|c|c|c|c|c|c|}
\hline & \multicolumn{6}{|c|}{ Formas parasitarias } \\
\hline & Tamaño promedio $(\mu)$ & Cruda & Primaria & Secundaria & Terciaria & \% Remoción total \\
\hline Entamoeba coli * & 25 & $15,50 \mathrm{a}$ & $4,37 a b$ & $3,00 \mathrm{a}$ & $0,50 a b$ & 96,77 \\
\hline Giardia duodenalis & 10 & $13,25 a b$ & $5,25 \mathrm{a}$ & $3,75 \mathrm{a}$ & 0,62 bc & 95,32 \\
\hline Taenia sp. & 35 & $8,00 \mathrm{bc}$ & $1,87 \mathrm{bc}$ & $0,63 b$ & $0,00 \mathrm{c}$ & 100,00 \\
\hline Rodentolepis nana & 45 & $5,00 \mathrm{c}$ & $0,88 \mathrm{c}$ & $0,13 b$ & $0,00 \mathrm{c}$ & 100,00 \\
\hline Ascaris lumbricoides & 60 & $14,25 \mathrm{a}$ & $3,00 \mathrm{abc}$ & $1,37 b$ & $0,18 b c$ & 98,73 \\
\hline Trichurus trichiura & 50 & $13,00 a b$ & $3,75 \mathrm{ab}$ & $1,37 b$ & $0,13 c$ & 99,00 \\
\hline Prueba de Fisher & - & 11,94 & 6,59 & 17,53 & 11,84 & - \\
\hline Probabilidad & - & 0,00 & 0,0001 & 0,00 & 0,00 & - \\
\hline
\end{tabular}

*) Especie no patogénica. Letras minúsculas iguales en sentido vertical indican que los promedios de formas parasitarias fueron estadísticamente iguales. Prueba de Tukey (SPSS versión 7,5).

En todos los casos no se observaron diferencias significativas a través del tiempo entre el promedio de formas parasitarias encontradas y las cuatro fechas de evaluación en cada una de las cuatro etapas de tratamiento (Tab. III).

Tabla III. Valores del estadístico $\mathrm{F}$ y niveles de probabilidad $(\mathrm{P})$ entre las cuatro evaluaciones realizadas entre septiembre y octubre del 2000 en cada uno de los puntos de muestreo de la Laguna de estabilización por ambas técnicas de concentración.

\begin{tabular}{lccccc}
\hline \multirow{2}{*}{ Fases del tratamiento } & \multicolumn{2}{c}{ Flotación de Faust } & & \multicolumn{2}{c}{ Sedimentación de Ritchie } \\
\cline { 2 - 3 } \cline { 5 - 6 } & $F$ & $P$ & & $F$ & $P$ \\
\hline Cruda & 0,01 & 0,99 & & 0,51 & 0,67 \\
Primaria & 0,26 & 0,84 & & 2,29 & 0,11 \\
Secundaria & 0,38 & 0,76 & & 0,43 & 0,73 \\
Terciaria & 0,32 & 0,80 & & 0,35 & 0,78 \\
\hline
\end{tabular}

Mediante ambas técnicas de concentración de formas parasitarias (Faust y Ritchie) se encontraron diferencias altamente significativas en el número total de formas parasitarias en cada una de las cuatro fases del tratamiento de las aguas residuales domésticas de la laguna de estabilización en San Martín de Porres, Lima, Perú. Siendo el promedio de formas parasitarias del crudo $>$ laguna primaria $>$ laguna secundaria > laguna terciaria (Tab. IV). Según la prueba de t de Student para datos pareados en cada una de las fases del tratamiento no se observaron diferencias en el número de formas parasitarias intestinales concentradas por ambas técnicas parasitológicas ( $\mathrm{t}=0,80, \mathrm{p}=0,43, \mathrm{n}=16)$.

La tabla $\mathrm{V}$, nos muestra la eficiencia de la remoción promedio de las formas parasitarias encontradas en las fases del tratamiento y las técnicas de concentración utilizada. Siendo el porcentaje de remoción en orden ascendente de la siguiente manera: Primaria a secundaria $>$ cruda a primaria $>$ secundaria a terciaria $>$ cruda a terciaria. 
Tabla IV. Número de formas parasitarias totales $\mathrm{L}^{-1}$ encontradas en cada una de las dos técnicas de concentración parasitológica para cada una de las cuatro fases del tratamiento de aguas residuales domésticas en una Laguna de estabilización en Lima, Perú.

\begin{tabular}{lcc}
\hline \multirow{2}{*}{ Fases del tratamiento } & \multicolumn{2}{c}{ Número de formas parasitarias totales $\mathrm{L}^{-1}$} \\
\cline { 2 - 3 } & Técnica de Faust & Técnica de Ritchie \\
\hline Cruda & $62,50 \mathrm{a}$ & $69,00 \mathrm{a}$ \\
Primaria & $21,62 \mathrm{~b}$ & $19,12 \mathrm{~b}$ \\
Secundaria & $10,37 \mathrm{c}$ & $10,25 \mathrm{c}$ \\
Terciaria & $1,56 \mathrm{~d}$ & $1,43 \mathrm{~d}$ \\
Prueba de Fisher & 1034,00 & 133,82 \\
Probabilidad & 0,00 & 0,00 \\
\hline
\end{tabular}

Letras minúsculas iguales en sentido vertical indican que los promedios de formas parasitarias fueron estadísticamente iguales. Prueba de Tukey (SPSS versión 7,5).

Tabla V. Eficiencia en la remoción de las formas parasitarias encontradas en cada una de las dos técnicas de concentración parasitológica en las fases de tratamiento de aguas residuales domésticas en una Laguna de estabilización en Lima, Perú.

\begin{tabular}{lcc}
\hline & \multicolumn{3}{c}{$\%$ de remoción total } \\
\cline { 2 - 3 } Fases del tratamiento & Técnica de Faust & Técnica de Ritchie \\
\hline Cruda a Primaria & 66,46 & 72,28 \\
Primaria a Secundaria & 50,29 & 46,40 \\
Secundaria a Terciaria & 84,93 & 85,97 \\
Cruda a Terciaria & 97,48 & 97,91 \\
\hline
\end{tabular}

\section{DISCUSIÓN}

MALICKI et al. (2001) han señalado que es importante el control parasitológico de las aguas residuales domésticas para su posterior empleo en irrigación y fertilización de cultivos agrícolas y ornamentales (CIFUENTES et al. 2000). Nuestros resultados indican que en la fase final del tratamiento de las lagunas de estabilización, en la etapa terciaria, aún el número de formas parasitarias sobrepasa el valor límite de $1 \mathrm{~L}^{-1}$ (Tab. IV), según las normas estándares en la Ley general de aguas en el Perú (Decreto Ley 17752), así como en las normas vigentes de otras latitudes. Sin embargo, si somos estrictos en lo que se considera como formas parasitarias patogénicas, debería excluirse a $E$. coli, que es considerado un no patogénico para el ser humano, en este caso el número de formas parasitarias patogénicas, por la técnica de flotación de Faust sería de 0,93 y también lo mismo, por la técnica de sedimentación de Ritchie de 0,93 (Tabs I, II, IV). De esta manera, ya no sobrepasa los valores límites máximos permisibles, reduciéndose de esta forma el riesgo para la salud humana al reusar dichas aguas residuales desde la óptica parasitológica.

Varios autores han señalado la importancia de la estandarización de las técnicas de conteo de quistes de protozoos y de los huevos de helmintos, como la sedimentación, la desorpción, la centrifugación y la flotación (MALICKI et al. 2001; NELSON \& DARBY 2001; PAULINO et al. 2001), pues debido a la técnica cuantitativa parasitológica empleada, se pudieran obtener resultados diversos, 
pudiendo observarse variaciones entre 30 al $75 \%$ en los resultados finales. Sin embargo, en este estudio, al compararse las dos técnicas de concentración con protocolos diferentes, una de flotación y otra de sedimentación se encontraron como promedio estadísticamente el mismo número de formas parasitarias (Tab. IV). En el caso de las siete especies de formas parasitarias encontradas, solo tres fueron comunes a ambos métodos de concentración parasitológica (Tabs I y II). Además, los quistes protozoos estuvieron mejor representados en el método de flotación de Faust (75\%), en contraste en la técnica de sedimentación de Ritchie, más diversos estuvieron los huevos de helmintos $(66,66 \%)$ (Tabs I y II). Otros autores han encontrado este mismo patrón (BOUHOUM \& SCHWARTZBROD 1989; MALICKI et al. 2001).

Aunque en general el tamaño de la forma parasitaria no estuvo relacionado linealmente con el porcentaje de remoción total, sino solo una relación logarítmica por el método de sedimentación. Se puede observar en un menor porcentaje de remoción total para los quistes de protozoos en comparación con los huevos de helmintos intestinales (Tabs I y II). Además, la eficiencia en la remoción de formas parasitarias se pudiera encuentra influenciada por la temperatura y por el tiempo de retención de las aguas residuales en cada una de las fases del tratamiento (MARA \& Silva 1986; PAUlino et al. 2001). En nuestro estudio la temperatura ambiental no varió significativamente entre las cuatro evaluaciones realizadas.

Por otro lado, se puede notar la mayor importancia del protozoo $G$. duodenalis en la laguna terciaria en comparación con el nemátodo A. lumbricoides (Tabs I y II). SEMENAS et al. (1999) muestran que el quiste del protozoo G. duodenalis y los huevos del nemátodo A. lumbricoides son dos formas parasitarias muy comunes de encontrar en las aguas residuales domésticas, debido a la característica cosmopolita de ambas formas parasitarias. Ambas especies presentan periodos de supervivencia bastante altos. En una recopilación de la caracterización parasitológica de las aguas residuales principalmente en Lima, Perú realizada por AurAzo (1996), se ha observado que $G$. duodenalis, E. coli y A. lumbricoides son las formas parasitarias más frecuentes.

De las cinco categorías de división de las infecciones causadas por los agentes patógenos por su forma de transmisión al medio ambiente indicados por SEMENAS et al. (1999), se han registrado tres categorías en nuestro estudio. Así, cuatro formas parasitarias pertenecientes a la categoría I: G. duodenalis, E. coli, I. belli e R. nana; dos a la categoría III: A. lumbricoides y T. trichiura y finalmente uno en la categoría IV: Taenia sp.

AGRADECIMIENTOS. A Celeste Miraval, Fernando Sernaqué y a Rubio Pavel por su colaboración en la toma de las muestras de aguas residuales. A los laboratorios de la Universidad Nacional Federico Villarreal por el apoyo en la presente Investigación. A Víctor Maldonado. Jefe de las Lagunas de Oxidación de San Martín de Porres, quien nos facilitó la toma de las muestras de aguas residuales. 


\section{REFERENCIAS BIBLIOGRÁFICAS}

APHA (American Public Health Association). 1995. Standard methods for the examination of Water and Wastewater. Washington, D.C., APHA, $18^{\text {th }} \mathrm{ed}$., $1100 \mathrm{p}$.

Aurazo, M. 1996. Caracterización de las aguas residuales: Aspectos parasitológicos. III Curso "Lagunas de estabilización”. Teoría, Diseño, Construcción, Operación, evaluación y mantenimiento. Lima, OPS/CEPIS, 6p.

AYres, M. \& D. MARA. 1997. Análisis de aguas residuales para su uso posterior en agricultura. Manual de Técnicas parasitológicas y bacteriológicas de laboratorio. Geneva, OMS, $31 \mathrm{p}$.

BouHoum, K. \& J. SCHWARTZBROD. 1989. Quantification of helminth eggs in waste waster. Zentralbl. Hyg. Umweltmed, Berlin, 188: 322-330.

Bush, O.A.; K.D. LAFFERTY; J.M. LotZ \& A.W. ShostaK. 1997. Parasitology meets ecology on its own terms: Margolis et al. Revisited. Jour. Parasitol., Washington, D.C., 83: 575-583.

Carrington, E.G.; E.B. PikE; D. Auty \& R. Morris. 1991. Destruction of faecal bacteria, enteroviruses and ova of parasites in wastewater sludge by aerobic thermophilic and anaerobic mesophilic digestion. Water Sci. Tech. Amsterdam, 24: 377-380.

Chavez, V.; P. Huapaya; Y. Espinoza; A. Huamán \& D. Kanashiro. 1994. Prevalencia de enteroparásitos y desnutrición infantil en un Centro Educativo del distrito del Rímac - Lima (1993). Rev. Per. Med. Trop. U.N.M. S.M., Lima, 8: 81- 85.

Cifuentes, E.; U. Blumental; G. Ruiz-Palacios; S. Bennett \& M. Quigley. 2000. Health risk in agricultural villages practicing wastewater irrigation in central Mexico: perspectives for protection. Schriftenr Ver Wasser Boden Lufthyg, Berlin, 105: 249-256.

Daniel, W.W. 1993. Bioestadística: Base para el análisis de las Ciencias de la Salud. Limusa. México, D.F., $3^{\text {ra }}$ ed., 667 p.

DE LOS Rios, J.C.Z. 1998. Plantas de tratamiento de aguas residuales y usos no convencionales. Revista "Saneamiento y Medio Ambiente" CISA-CIP (Perú), Lima, 4: 11- 13.

ESPINOZA, E.P. 1998. Análisis de aguas residuales para plantas de tratamiento. Revista "Saneamiento y Medio Ambiente" CISA- CIP (Perú), Lima, 4: 3-6.

Habbari, K.; A. Tifnouti; G. Bitton \& A. Mandil. 2000. Geohelminth infections associated with raw wastewater reuse for agricultural. Parasitol. Int., Amsterdam, 48: 249-254.

Howard, S.C.; C.A. Donnell \& M.S. Chan. 2001. Methods for estimation of association between multiple species parasite infection. Parasitology, Cambridge, 122: 233-241.

Jakubowski, W.; J.L. Sykora; C.A. Sorber; L.A. Casson \& P.D. Gavaghan. 1991. Determining giardiasis prevalence by examination of sewage. Water Sci. Tech., Amsterdam, 24: 173-178.

MaLicki, J.; A. Montusiewicz; A. BIEgANOwSKI. 2001. Improvement of counting helminth eggs with internal standard. Water Res., Amsterdam, 35: 2333-2335.

MARA, D.D. \& S.A. SiLVA. 1986. Removal of intestinal nematode eggs in tropical waste stabilization ponds. Jour. Trop. Med. Hyg., Oxford, 89: 71-74.

NELSON, K.L. \& J.L. DARBY. 2001. Inactivation of viable Ascaris eggs by reagents during enumeration. Appl. Environ. Microbiol., New York, 67: 5453-5459.

OMS. 1992. Métodos básicos de laboratorio en parasitología médica. Ginebra, OMS, 116p.

PAulino, R.C.; E.A. CASTRo \& V. ThOMAZ-SocCOL. 2001. Helminth eggs and protozoan cysts in sludge obtained by anaerobic digestion process. Rev. Soc. Bras. Med. Trop., Uberaba, 34: 421-428.

Rose, J.B. \& G.C. GERBA. 1991. Use of risk assessment for development of microbial standards. Water Sci. Tech., Amsterdam, 24: 29-34.

Rose, J.B.; H. Darbin \& G. C. Gerba. 1988. Correlations of the protozoa, Cryptosporidium and Giardia with water quality variables in a watershed. Water Sci. Tech., Amsterdam, 20: 271-276.

SÁNCHEZ, M.C.A. 1991. Estudio de factibilidad para el tratamiento de aguas residuales mediante lagunas de oxidación. Lima, CEPIS, OPS/OMS Ed., 336p.

Semenas, L.; N. Brugni; G. Viozzi \& A. Kreiter. 1999. Monitoring of parasites in domestic sewage.

Rev. Saúde Publica, São Paulo, 33: 379-384.

Sencico (Servicio Nacional de Capacitación para la Industria de la Construcción). 1996. Proyecto de

Revta bras. Zool. 19 (4): 1033 - 1041, 2002 
Norma Técnica de edificación S.010 Planta de tratamiento de aguas residuales. Lima, SENCICO, 72p.

Shuval, H.I.; Y. Perez \& F. Badri. 1985. Epidemiological evidence for helminth and cholera transmission by vegetables irrigated with wastewater; Jerusalem a case study. Water Sci. Tech., Amsterdam, 17: 433-442.

Smrth, H.V. 1999. Detection of parasites in the environment. Parasitology, Cambridge, 117: 113-141.

Toranzos, G.A. 1991. Current and possible alternate indicators of fecal contaminations in Tropical waters: a short review. Environ. Toxicol. Water Qual., New York, 6: 121-130.

VARGAS, M.C. 1988. Aspectos microbiológicos y biológicos de las guías sobre calidad de agua potable. Manual del curso taller sobre control de calidad analítica de Laboratorios. Lima, SENAPA, 23p.

WATSOn, D.C.; M. SATCHWEel \& C.E. JonES. 1983. Study of the prevalence of parasitic helminth eggs and cyst in sewage sludges disposed of to agricultural land. Jour. Water Poll. Control Fed., Washington, D.C., 3: 3285- 3289.

ZAPATA, S.M.; F.T. TORRICO \& M.A. M. SOLANO. 1991. Estudio microbiológico de aguas residuales hospitalarias de la ciudad de Cochabamba. Universidad Mayor de San Simón (Cochabamba, BO). Facultad de Medicina. Salud Ambiental, un desafío hacia el año 2000. La Paz, ABIS, 12p.

ZAR, J.H. 1996. Biostatistical analysis. New Jersey, Prentice-Hall Inc., $3^{\text {th }}$ ed., 662p.

Recebido em 13.III.2002; aceito em 01.X.2002. 